Verlag von VEIT \& COMP. in Leipzig.

\title{
Ueber Tuberculose.
}

Die Verbreitung der Tuberkelbacillen ausserhalb des Körpers. Die Sterblichkeitsverhältnisse in den Krankenpflegeorden.

Von

\section{Dr. Georg Cornet,}

prakt. Arzt in Berlin und Reichenhall.

Mit einem Anhange:

Die von Behörden, Kurorten u. s. w. in letzter Zeit erlassenen Verordnungen in Bezug auf die Prophylaxis der Schwindsucht.

Mit 4 Figuren im Text.

gr. 8. 1890. geh. 4 A.

\section{Grundriss der Hygiene.}

Für Studirende und praktische Ärzte, Medieinal - und Verwaltungsbeamte.

\section{Von \\ Dr. C. Flügge,}

o. 0. Professor der Hygiene u. Director des hygienischen Instituts an der Universitatt Breslau.

Mit Abbildungen im Text und zwei Tafeln.

gr. 8. 1889. geh. $11 ~ \%$; geb. in Ganzleinen $12 \%$.

\section{Lehrbuch der Geburtshülfe} einschliesslich

der Pathologie und Therapie des Woohenbettes.

Für praktische Arzte und Studirende.

\section{Von \\ Dr. F. Winckel,}

Professor d. Gynäkologie u. Director d. Königl. Frauenklinik a. d. Univ. Mănchen.

Mit 188 Holzschnitten im Text.

Lex.-8. 1889. geb. $22 \not{A} ;$ geb. $24 \mathscr{A} 50 \not 9$. 
Verlag von VEIT \& COMP. in Leipzig.

Chirurgisch-anatomisches Vademecum

für Studierende und Ärzte

Prof. Dr. W. Roser.

Achte, sorgfältig umgearbeitete Auflage,

besorgt von

Dr. Karl Roser.

Mit 189 Abbildungen.

8. 1890. Gebunden in Ganzleinwand 6 \%.

Grundriss

$$
\text { der }
$$

Physiologie des Menschen für Studirende und Aerzte.

Von

Prof. Dr. J. Steiner.

Fünfte; verbesserte Auflage.

Mit zahlreichen in den Text eingedruckten Holzschnitten.

gr. 8. 1890 . geh. $9 \mathscr{K}$; geb. $10 \mathscr{K}$.

\section{Grundzage einer \\ allgemeinen Pathologie der Zelle. \\ Vorlesungen, \\ gehalten an der $K$. Universität Warschau \\ S. M. Lukjanow,}

o. ö. Professor der allgemeinen Pathologie an der Kajs. Universitat Warachau.

gr. 8. 1891. geh. $7.450 \not 7$. 


\title{
Verlag von VEIT \& COMP. in Leipzig.
}

\section{Lehrbuch}

\section{allgemeinen und speciellen Chirurgie}

\author{
einschliesslich
}

der modernen 0perations- und Verbandlehre. Von

\section{Dr. Hermann Tillmanns, \\ Professor an der Universitat Lieipzig. \\ Zwei Bände.}

Roy.-8. geh. $40 \AA ⿻$

\section{Erster Band.}

Lehrbuch der allgemeinen Chirurgie.

Allgemeine 0perations- and Verband-Technik.

Allgemeine Pathologie und Therapie.

Mit 337 Abbildungen im Text.

1888. geh. $12 \mathscr{K}$; geb. in Halbfr. $14 \mathscr{A} 50$ g\%.

\section{Zweiter Band.}

Lehrbuch der speciellen Chirurgie.

\section{Mit 791 Abbildungen im Text.}

1891. geh. $28 \%$; geb. in Halbfr. $31 \%$. 
R. Hagen, Anleiting. 6. Auflage.

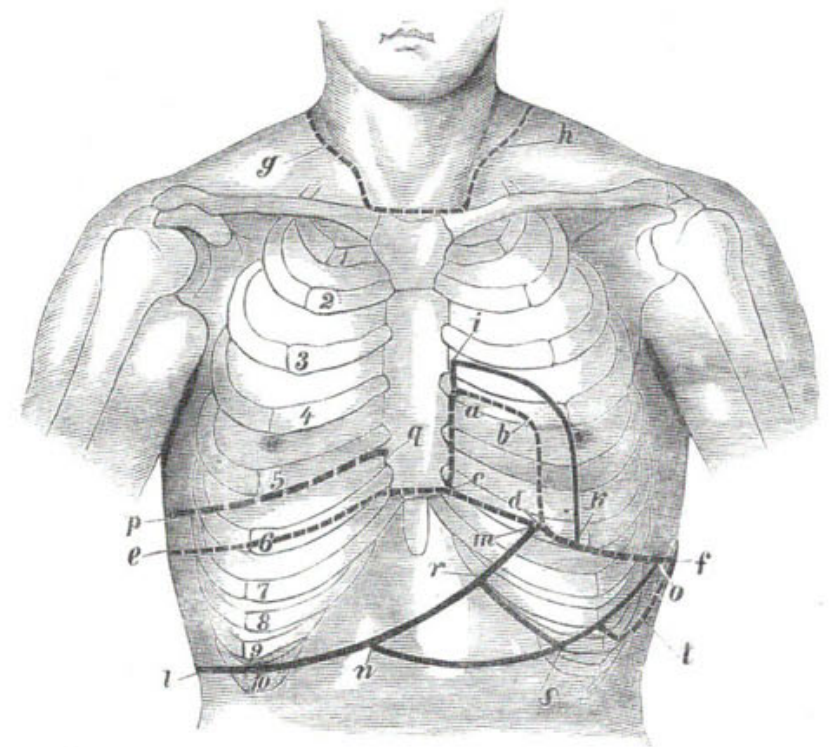

Die percutorischen Grenzen der Lungen, des Herzens, der Leber und des Magens von vorn. (Nach WeIL.)

$a b$ obere, $a c$ rechte, $c d$ untere, $b d$ linke Grenze der absoluten Herzdämpfung; $c e$ untere Grenze der rechten, $d f$ untere Grenze der linken Lunge. $g h$ obere Lungengrenze, a $i k d$ Grenzen der relativen Herzdämpfung; $l m$ unterer Rand der Leberdämpfung; $p q$ obere Grenze der relativen Leberdämpfung; $n \boldsymbol{8 0}$ untere Magengrenze bei mittlerer Ausdebnung des Magens; $t$ vorderes Ende der Milz; r sf $m$ 'TRAUBE's halbmondformiger Raum. 


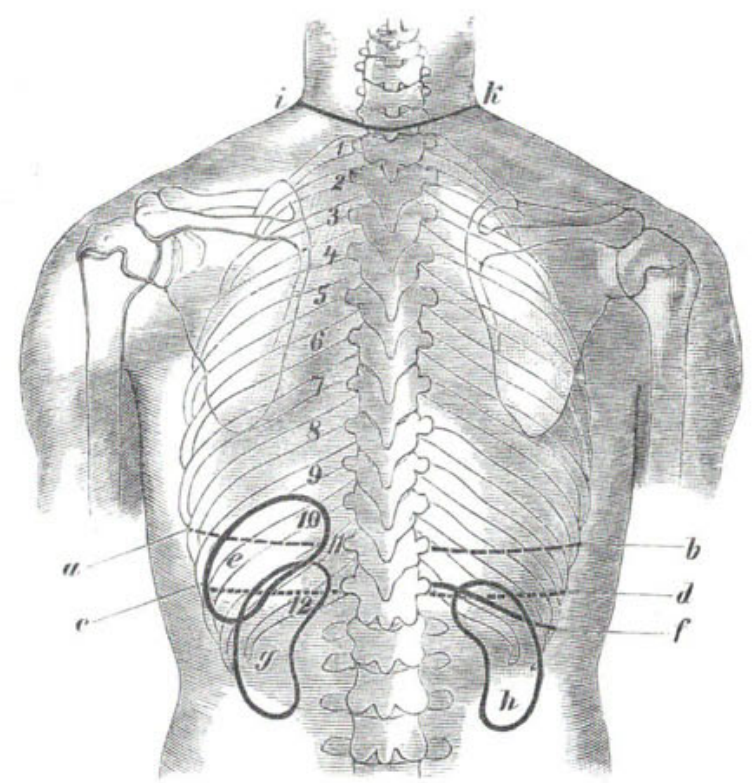

Lage der Brust und Bauchorgane von hinten. (Nach Loschka.)

$a b$ unterer Lungenrand; $c d$ untere Pleuragrenze; $i k$ obere Lungengrenze; $e$ Milz; $f$ unterer Leberrand; $g$ linke Niere $h$ rechte Niere. 


\title{
Verlag von VEIT \& COMP. in Leipzig.
}

\section{E H R B U C H}

der

speziellen

\section{Pathologie und Therapie} mit

besonderer Berücksichtigung der Therapie.

\author{
Für Studierende und Ärzte \\ von \\ Dr. Theodor v. Jürgensen,
}

o. ö. Professor der Medlzin und Vorstand der Poliklinik an der Universität Tübingen.

\section{$=$ Zwoite, $=$ verbesserte und vermehrte Auflage.}

Ein Band von 50 Bogen Roy.-8. geh. $15 \varkappa$, in Halbfranz geb. $17 \mathcal{k}$.

„Das Buch ist kurz und gut, möge der Leser selbst entscheiden, ob ich viel gesagt. Mit diesen Worten schloss Professor Wintennitz die Besprechung der ersten Auflage des vorliegenden Buches in der Wiener medizinischen Presse. Der Leser hat entschieden und den Ausspruch von Winternitz vollkommen bestätigt. Das beweist wohl das Erscheinen der zweiten Auflage in einem relativ kurzen Zeitraum nach der ersten.

Unter den kurzen Lehrbüchern der speziellen Pathologie und Therapie nimmt sicherlich das vorliegende die erste Stelle ein. Die auf reicher Erfahrung fussende, knappe, dabei aber erschöpfende und lebendige Darstellung verleiht dem Buche einen besonderen Reiz und Werth und schützt es vor der mit Recht gefürchteten Lehrbuch. Trockenheit. Dass die neueren Forschungsresultate mit kritischem Auge in die Darstellung mit einbezogen wurden, ist selbstverständlich. - - -

Gegenüber der ersten Auflage ist die zweite nicht nur durch die Aufnahme der neueren Forschungsergebnisse, sowie durch Erweiterung mancher zu karg behandelter Abschnitte, wie z. B. Peritonealtuberculose, sondern anch durch Aufnahme ganz nezer Kapitel bereichert." - -

Wiener med. Presse. 1890. No. 40. 


\section{Anleitung \\ zur \\ klinischen Untersuchung und \\ Diagnose.}

\section{Ein Leitfaden für angehende Kliniker von}

Dr. med. Richard Hagen, a. o. Professor der Medicin an der Universitat Leipzig.

Sechste, verbesserto and vermehrte Anflage. Mit 25 Abbildungen im Text und elner Tafel.

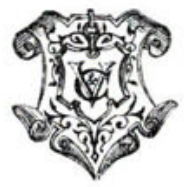

Leipzig,

Verlag von Veit \& Comp. 1891. 
Das Recht der Heraugabe von Uebersetzungen in fremde Sprachen vorbehalten.

Druck ron Metzger \& Wittig in Leiprig. 